\title{
HEAL Project Aims to Regenerate Human Limbs by 2030
}

Published online: 7 December 2015

(C) The Regenerative Engineering Society 2015

Recently, the Institute for Regenerative Engineering (IRE) at the University of Connecticut (UConn) Health announced the launch of the Hartford Engineering A Limb (HEAL) initiative, which aims to "regenerate a new human joint in 7 years and regenerate an entire limb in 15 years." About 25 UConn researchers will be involved in this HEAL project, including Professors Cato Laurencin, Lakshmi Nair, and Yusuf Khan. Also, scientists and clinicians from around the world will participate in the HEAL project, including research teams at UC Irvine, Harvard University, Columbia University, and SASTRA University in India. The researchers will combine cutting-edge technologies across tissue engineering, bioengineering, and regenerative medicine, which will be the first international collaboration for the knee and the limb regenerative engineering.

For leading this new interdisciplinary research program of regenerative engineering, Dr. Cato Laurencin has won a National Institutes of Health (NIH) Pioneer Award, which according to the NIH is for "scientists of exceptional creativity who propose pioneering and possibly transforming approaches to addressing major biomedical or behavioral challenges that have the potential to produce an unusually high impact on a broad area of biomedical or behavioral research." The HEAL project and IRE are also supported by the grant award from the National Science Foundation for Emerging Frontiers in Research and Innovation, funding from the Raymond and Beverly Sackler Center for Biomedical, Biological, Physical and Engineering Sciences at UConn Health, and the State of Connecticut.

Over the past 20 years, Dr. Laurencin and his colleagues have developed innovative technologies to create component tissues, such as bone, cartilage, ligaments, tendon, skin, nerve, and blood vessels, which have been successfully implanted in human clinical trials. Dr. Laurencin's strategy for regenerative engineering focuses on the engineering biomimetic tissues that can grow after being integrated into patients rather than making robotic limbs. Now, inspired from salamanders and newts which can regenerate their limbs within few weeks after amputation, they challenge to engineer a complex musculoskeletal system to help patients who have lost limbs or damaged nerve due to cancers, diabetes, infections, trauma accidents, and congenital defects.

By utilizing regenerative engineering technology, the team will develop integrated graft systems, such as osteochondral, osteoligamentous, muscle/tendon, and vascularized tissues. Also, peripheral nerves will be integrated into the tissues to generate additional tissues. The team will apply biological, electrical, and biomechanical cues from developmental biology for the regeneration and integration of complex tissues and limb. The HEAL project aims to revolutionize the treatment of musculoskeletal tissues and will generate knowledge that can extend into regeneration of other tissue systems.

\section{In Vivo Cellular Reprogramming by Nanoneedles}

\section{Nanoneedles can access in vivo intracellular targets to deliver biomolecules with an efficiency greater than $90 \%$, inducing sustained neovascularization and an increase in blood perfusion in muscle tissue for regenerative engineering}

Regenerative engineering is a convergence of tissue engineering with advanced materials science, stem cell science, and 
developmental biology, which aims to repair complex tissues [1]. In this regard, regenerative engineering can enhance complicated tissue healing by inducing vascularization, which can be done by reprogramming of cells through minimally invasive gene delivery systems. Here, we introduce an innovative approach of nanoneedle material to demonstrate one example of regenerative engineering.

The recent development in high-aspect-ratio nanoneedle structures has enabled single cells to be monitored and small biomolecules to be directly delivered into the targeted area of intracellular space [2-4]. During penetration of the cellular membrane with nanoneedles, cells can function normally as minimal intrusion occurs. Now, writing in Nature Materials, Ennio Tasciotti, Molly Stevens, and colleagues from Imperial College London and Houston Methodist Research Institute in the USA reported an innovative approach of applying nanoneedles in vivo to directly reprogram cells to induce tissue regeneration (Fig. 1) [5, 6]. The team showed that biodegradable silicon nanoneedles can co-deliver DNA and siRNA into the cytosol of cells with an efficiency greater than $90 \%$. As a result, neovascularization and blood perfusion were significantly enhanced in the selected tissue area. In addition, even with this early prototype, nanoinjection did not show any inflammation in muscle or skin and required only a very small incision level $(<1 \sim 2 \mathrm{~cm})$.

From hypodermic injection to nanoneedle injection, scale of the injection has been reduced to minimize tissue damage and enhance accuracy of targeting. Since the first patch was approved by the US FDA in 1979, transdermal delivery has been dramatically expanded to overcome biological barriers for effective drug delivery [7, 8]. Starting from the first generation of basic patches, the second- and third-generation transdermal delivery systems increased skin permeability and provided a driving force for drug transport into the skin by chemical enhancers and physical stimulation, respectively. Microneedles belong to the third-generation delivery system and have been successfully created as a painless microscale pathway to deliver drugs through the skin. As the scale of needles decreased into nanoscale, nanoneedles have enabled

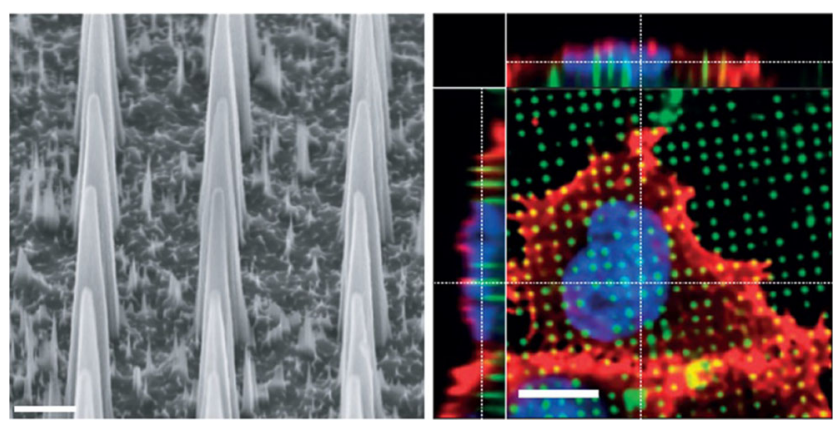

Fig. 1 Biodegradable silicon nanoneedles (left, scale bar $2 \mu \mathrm{m}$ ) and cells interfacing nanoneedles (right, scale bar $10 \mu \mathrm{m}$ ). Adapted with the permission of Macmillan Publishers Ltd. (Nature Materials) [5], copyright 2015 the efficient delivery of small hydrophilic biomolecules directly into the intracellular target by penetrating the cellular membrane.

To create silicon nanoneedles, Tasciotti and co-workers prepared a patterned silicon nitride masking layer on top of silicon wafers using photolithography. By metal-assisted chemical etching, silicon pillars were formed underneath the silicon nitride pattern and then redesigned into a conical needle shape by reactive ion etching. The prototype nanoneedles had $5 \mu \mathrm{m}$ length, $50 \mathrm{~nm}$ apical width, and $600 \mathrm{~nm}$ base diameter with densities of approximately 25 million needles $/ \mathrm{cm}^{2}$ on an $8 \times 8-\mathrm{mm}^{2}$ chip, providing an over 300 -fold increase in surface area compared to cylindrical nanowire structures [9]. The diameter of the nanoneedles was controlled by changing the duration of the plasma etching. To load nucleic acids on nanoneedles, the surface of the chips was silanized to become positively charged and attract negatively charged nucleic acids.

Nanoneedles had sufficient mechanical strength $(\sim 260 \mathrm{nN})$ for in vitro and in vivo applications, as the force experienced during penetration of a cell is known as $2 \mathrm{nN}$. On the other hand, silicon nanoneedles were biodegradable in physiological condition that their shape ultimately dissolved within $72 \mathrm{~h}$. Therefore, silicon nanoneedles had unique characteristics of being both robust enough to penetrate the cellular membrane and, at the same time, biodegradable to completely dissolve over time. When cells were penetrated by nanoneedles, they maintained physiologic metabolic activity and normal proliferation level. There was no leakage of intracellular material. In addition, the team evaluated the efficiency of nanoinjection of VEGF165 plasmid DNA, which is a master angiogenic gene, in an in vivo mouse model. A small incision was made in the mouse model and VEGF-loaded nanoneedles were implanted in the muscle and compared with the group of direct injection of VEGF. Notably, only nanoinjected muscle exhibited much higher neovascularization with 6-fold increase in blood perfusion and the number of nodes in the vasculature, while the direct injection of the VEGF group did not show significant enhancement in neovascularization.

The major innovation that Tasciotti and co-workers achieved is that they have made a nanoneedle platform with high transfection efficacy of loading and releasing nucleic acids directly inside cells in dynamic in vivo conditions which actually induced effective neovascularization. This approach extended the previous single-cell level of nanoneedle application into simultaneous reprogramming of cells in a complex tissue level as a regenerative engineering. Also, nanoneedles showed high potential as a non-immunogenic delivery platform for small biomolecules to other tissues as well. Conceivably, nanoneedles can be used with other delivery systems to enhance the accuracy of targeting and become multifunctional systems for sensing, stimulating, and monitoring of cells in vivo, to facilitate development of regenerative engineering approaches. 


\section{Active Tumor Targeting by Homing Lymphocytes}

In vivo homing of autologous lymphocytes can deliver anticancer drugs to poorly accessible tumor-bearing lymph nodes with high efficiency to result in tumor reduction and tissue regeneration

Development in active cell-mediated delivery, which has grown in concurrence with tissue engineering, material science, stem cell science, and biological pathway analysis, has led to repair of complex tissues, as a facet of regenerative engineering [1]. Here, we introduce a novel cancer chemotherapy treatment that effectively targets disseminated tumors by using tissue-homing lymphocytes as a living drug delivery agent.

Lymphoma is a cancer developed in the lymphatic system, which can lead to metastasis in multiple organs as it is derived from the lymphatic cells that circulate along the lymphoid organs. Once the lymphoma enters into the lymph nodes, it becomes difficult to treat with general cancer chemotherapy. The permeation of the drug throughout the blood vessels and tumor is often hindered by rapid metabolism, excretion, and biological barriers and thus inevitably requires excess dosage to induce the proper level of an anticancer therapeutic effect $[10,11]$. Even with the support of current advanced drug delivery systems using nanoparticles, issues such as low efficiency of targeting tumor cells in lymph nodes arose, as nanoparticles were often trapped outside the tumor while their delivery pathways were mostly limited to the presence of the leaking vasculature. To augment the therapeutic efficacy of drug delivery while reducing toxic side effects of the drugs, writing in Science Translational Medicine, Darrell J. Irvine and colleagues from Massachusetts Institute of Technology designed and developed active targeting chemotherapy on cancer cells by using nanoparticle-carrying $\mathrm{T}$ cells with engineered homing receptors to access into tumor-bearing lymph sites (Fig. 2) [12]. The team expanded autologous polyclonal $\mathrm{T}$ cells ex vivo, functionalized their surface to carry drug-loaded nanocapsules, and transferred carrier T cells in a murine model of disseminated lymphoma. This "Trojan horses" strategy of nanoparticle-decorated T cells to actively target hidden tumor cells has increased potency of the drug more than 40-fold greater than dosing free drug, enhancing overall tumor recovery in vivo.

Since cancer has been one of the most critical causes of human death, many treatments have been investigated, including surgical intervention, radiation, and chemotherapeutic drugs [13]. To minimize toxic adverse effects of anticancer drugs by enhancing the efficiency of tumor targeting, drug delivery has been rapidly developed by the integration of nanotechnology and advanced organic/inorganic materials. Current chemotherapeutics target tumor cells either passively by extravasation of nanocarriers through the leaky vasculature based on enhanced permeability and retention (EPR) effect or actively by conjugating nanocarriers with molecules that can bind to target tumor cells [14]. To increase circulating time in blood and to enhance targeting efficiency, cell-mediated drug delivery systems using circulating cells, such as red blood cells, white blood cells, and stem cells, have recently gained increasing attention due to their disease tracking capacities and homing properties [15]. Among circulating cells, T lymphocytes have generated promising results as carriers to deliver chemotherapy agents to treat hematologic malignancies $[16,17]$.

In the recent literature, Darrell J. Irvine and colleagues used polyclonal $\mathrm{T}$ cells which can be obtained with large numbers ( 250 to 500 million) from a single leukapheresis and quickly expanded ( $\sim 5000$-fold), whereas tumor-specific T cells can be only isolated from patients. Nanocapsules were synthesized by divalent cation-mediated fusion of anticancer drugs, SN38-loaded anionic bilayers, and subsequent stabilization by the formation of covalent crosslinks between these liposome walls to build multilamellar lipid capsules. After crosslinking, remaining maleimide groups on the surface of nanocapsules were conjugated with thiols of $\mathrm{T}$ cells and the residual maleimide groups were quenched by polyethylene glycol-thiol. Without maleimide groups, nanocapsules and T cells had minimal nonspecific interactions.
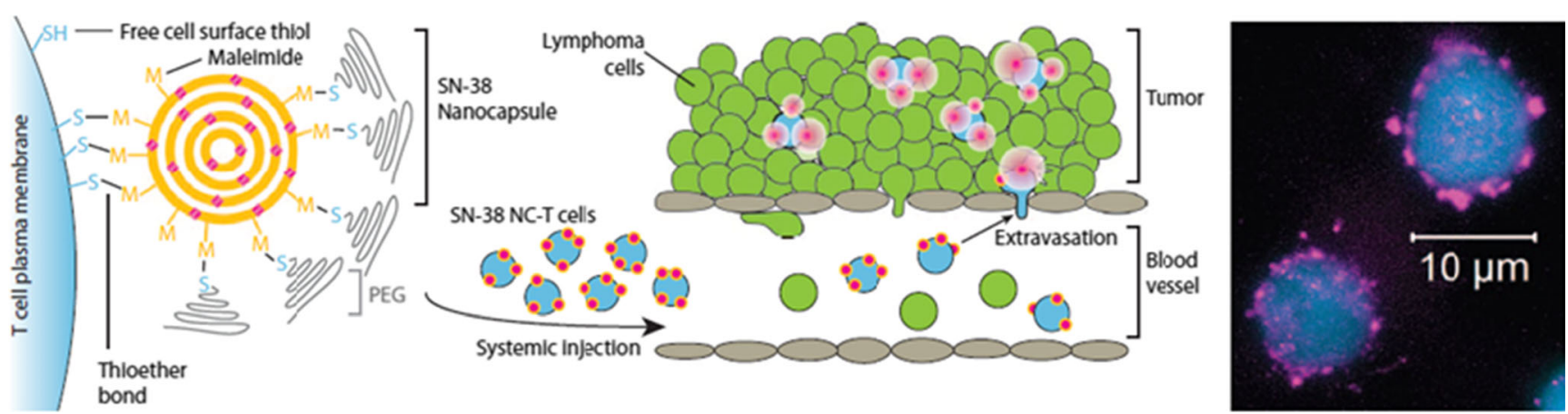

Fig. 2 Schematic of cell-mediated delivery of drug-loaded nanoparticles into poorly accessible tumors (left) and confocal microscopy image of T cells conjugated to nanocapsules (from Huang et al. 2015. Science Translational Medicine, 7:291 ra94; reprinted with permission from AAAS) 
The direct conjugation of SN-38-loaded nanocapsules on the cellular membrane did not cause toxicity or alter the functions of carrier cells. After the injection of nanocapsule-loaded T cells (NC-T cells) into a tumor-bearing mouse model, NC-T cells were dispersed throughout the tumor-bearing lymphoid organs. The concentration of SN-38 in tumor-bearing lymph nodes delivered by NC-T cells was 63-fold greater than that of free nanocapsules. As a result, animals treated with SN-38 NC-T cells exhibited a 60 -fold reduction of tumor burden. In addition, based on the result that a one-fourth dose of NC-T cells showed a similar level of tumor burden with ten times more concentrated free SN-38 dose, NC-T cell-mediated delivery had increased the potency of SN-38 by 40 -fold while it simultaneously lowered off-target toxicity.

The active targeting strategy developed by Darrell J. Irvine and colleagues, using tissue homing of autologous lymphocytes as chaperones for drug delivery, achieved a high efficacy of drug delivery to poorly accessible tumor sites. The efficiency of a drug-loaded cellular platform has a high potential to continuously increase by converging the ongoing progress of nanotechnology into a carrier design. In addition, autologous T cells can be easily obtained from the blood and expand into mass quantity. As T cells are involved in the cell-mediated immunity of most tissues, tissue homing of lymphocyte carriers can be applied into other organs as well by modifying homing ligands related to targeting of T cells. It is conceivable that these actively targeting pharmacytes will be applied to clinical trials to cure diseases and to regenerate tissues.

\section{Whitlockite, the Secondary Biocrystal for Bone Regeneration}

\section{Whitlockite, the second abundant mineral in human bone, can stimulate early osteogenic differentiation of mesenchymal stem cell and regenerate new bone tissues in vivo}

The recent development of nanotechnologies in material science with parallel advances in tissue engineering, stem cell engineering, and developmental biology have enabled the regeneration of complex tissues and biological systems [1]. Here, we present a novel bone-mimetic implant material at nanoscale as one representative example of regenerative engineering.

The two major biominerals in bone are hydroxyapatite (HAP: $\left.\mathrm{Ca}_{10}\left(\mathrm{PO}_{4}\right)_{6}(\mathrm{OH})_{2}\right)$ and whitlockite $(\mathrm{WH}$ : $\left.\mathrm{Ca}_{18} \mathrm{Mg}_{2}\left(\mathrm{HPO}_{4}\right)_{2}\left(\mathrm{PO}_{4}\right)_{12}\right)$, while $\mathrm{WH}$ can exist up to approximately $20 \mathrm{wt} \%$ in the inorganic part of bone [18]. However, despite its abundance in bone, research related to $\mathrm{WH}$ has been insufficiently conducted due to difficulty in its synthesis. As a result, WH has never been implemented in the clinical field and simultaneously, the nanoscale composition of bone has not been reflected in bone material. Only recently, a new gramscale synthetic method of pure-phase WH nanoparticles has been developed by Jang et al. [19]. Now, in the currently reported literature, Jang and colleagues designed a porous bone implant platform and evaluated the biocompatibility of $\mathrm{WH}$ in vitro and in vivo, for the first time (Fig. 3) [20]. From the calvarial defect of a rat model, the WH implant showed similar or better bone regeneration capabilities compared to the HAP implant. These new findings of WH implicate that the two major bone minerals have different biological roles in bone.

Calcium phosphates are the most widely used bone graft materials based on their similar composition with bone and excellent biocompatibilities. Among various calcium phosphates, HAP is the main biomineral in bone and the most commonly used bioceramic for bone material. However, when synthetic HAP is inserted in vivo, it hardly dissolves and thus remains for a long period of time. To provide biodegradability, bone implant materials are often mixed with highly soluble $\beta$ tricalcium phosphate ( $\beta$-TCP: $\left.\mathrm{Ca}_{3}\left(\mathrm{PO}_{4}\right)_{2}\right)$, which is the synthetic analog of WH. However, $\beta$-TCP has a high-temperature phase which can be produced above around $900{ }^{\circ} \mathrm{C}$ and thus does not exist in bone tissue, while its particle size becomes larger than the micrometer scale after a sintering process. In this respect, the composition of a current bone implant composed of a HAP/ $\beta$-TCP mixture has been inevitably limited to the micrometer scale, instead of reflecting the nanoscale composition of bone. In addition, implanted $\beta$-TCP is expected to dissolve and be replaced by regenerated bone as a transitional structure. Therefore, the development of advanced bone material in the nanoscale is required to create a bone-mimetic material that can directly harmonize with surrounding bone tissue immediately after implantation to induce rapid tissue regeneration.

Jang and colleagues synthesized WH nanoparticles in an acidic aqueous environment with an excess amount of $\mathrm{Mg}^{2+}$ to impede the growth of HAP. According to the previously reported precipitation conditions for WH [19], an anionic source (phosphoric acid) was slowly added into a cationic source (mixed solution of calcium hydroxide and magnesium hydroxide) which led to a decrease in $\mathrm{pH}$ from a basic condition to an acid condition, where the precipitation of a $\mathrm{WH}$ phase was favored [21]. For the in vitro study, a salt leaching method was used to form a 3D macroporous WH-embedded poly-L-lactic acid/poly lactic-co-glycolide scaffold to seed mesenchymal stem cells across the entire scaffold. In addition, for the in vivo study, polymethyl methacrylate polymer beads were incorporated into $\mathrm{WH}, \mathrm{HAP}$, and $\beta$-TCP particles to form spherical pores after sintering. Disk-type implants were fabricated by applying pressure in a cylindrical mold, and by crushing and sieving, 1-mm-sized homogeneous porous implants were obtained. The mechanical strength of the implants was equalized by controlling the sintering temperature of each bioceramic material. 
Fig. 3 WH nanoparticles synthesized on a gram scale (left), HRTEM image of 50-nm WH nanoparticles (middle), and a MicroCT image of a WH implant in a rat calvarial defect model, 6 weeks after insertion (right) Reproduced with permissions [20]. Copyright 2015, John Wiley \& Sons, Inc.
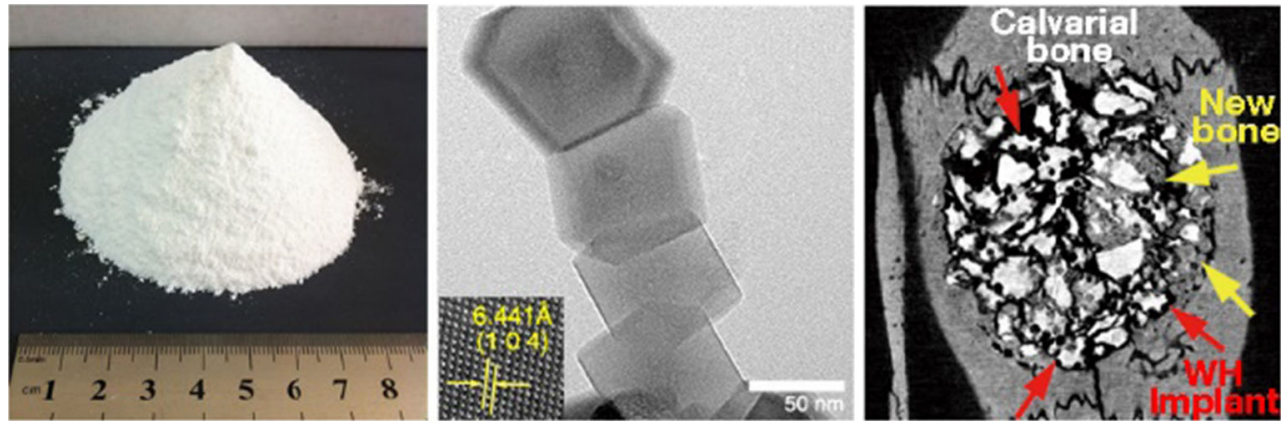

From the in vitro test, cells grown on the WH-reinforced composite scaffolds exhibited a dramatic increase in cellular gene expression level related to bone mineralization compared to the HAP-reinforced composite scaffolds. ALP, Col I, and Runx2 osteogenic gene expressions were increased approximately 25.3-fold, 36.9-fold, and 4.6-fold, respectively. After WH implants were implanted into a rat calvarial defect model, WH implants generated a higher amount of newly formed bone than HAP implants and $\beta$-TCP implants. Also, the presence of osteoblast-like cells around WH implants indicated that osteoprogenitor cells have been migrated for bone regeneration. Notably, WH implants showed intermediate resorbability between HAP implants and $\beta$-TCP implants.

Based on the recently developed synthetic method of WH nanoparticles, Jang et al. evaluated the biocompatibility of the secondary mineral of human bone for the first time and showed high possibility for its practical application. A high level of $A L P$ expressed from cells grown on a WH scaffold indicated participation of WH in the early phase of osteogenic differentiation, which well corresponded to the presence of a higher ratio of WH in younger aged and earlier stages of bone mineralization $[22,23]$. Being in vivo, WH implants regenerated a higher amount of bone tissues compared to currently used HAP and $\beta$-TCP implants in clinics. New approaches of utilizing the secondary bone mineral will also provide valuable opportunities to understand complex interplays among bone minerals, cells/proteins, and other tissues around bone tissue at nanoscale for the further development of regenerative engineering.

\section{Releasing Multiple Growth Factors from Wound Dressing}

\section{Multiple growth factor release from wound dressing can stimulate angiogenesis in chronic diabetic ulceration and induce rapid tissue regeneration in vivo}

Regenerative engineering aims to heal complex tissues and biological systems based on multidisciplinary approaches, including tissue engineering, advanced material science, stem cell science, and developmental biology [1]. In this sense, regenerative engineering can improve wound recovery, because complicated causative factors are involved through multiple tissues during wound development. Here, we introduce an interesting approach to fabricating a wound dressing platform, which contains a combination of growth factors.

One fourth of diabetic patients experience painful chronic foot ulcerations which often lead to amputation $[24,25]$. Unlike healthy wounds, chronic wounds that develop in diabetic patients are difficult to be repair, as release of growth factors and cytokines is disrupted [26, 27]. In this respect, there have been many clinical attempts to utilize exogenous growth factors for tissue repair in diabetic patients. However, until now, only a few growth factors have been translated into clinical wound dressings, while their efficacies and safeties are significantly limited. Now, in a paper recently published in $A d$ vanced Healthcare Materials, Paula T. Hammond and colleagues presented a novel approach to efficiently deliver multiple growth factors for diabetic ulcer healing, through mimicking the endogenous repair process of a healthy wound [28]. From the commercially available nylon wound dressings, they constructed drug-loaded multilayer films which contained vascular endothelial growth factor $165(V E G F)$ and plateletderived growth factor $\mathrm{BB}(P D G F-B B)$ to promote angiogenesis. This new flexible wound healing platform dramatically enhanced drug delivery efficiency by achieving a similar level to traditional delivery methods with its protein amount levels only less than 300 times and successfully formed granulation tissues in vivo.

Wound healing proceeds through several stages, including inflammation, cellular migration/proliferation, granulation tissue formation/degradation, and final tissue regeneration $[29,30]$. For the diabetic chronic wound, there are critical hindrance factors for wound recovery, such as peripheral artery disease, microvascular disease, and increased infection susceptibility caused by abnormal activities of cells and/or cytokines. In this respect, wound dressing should not only act as a passive protective barrier but also actively provide an optimal environment for cellular migration/proliferation and angiogenesis to stimulate tissue regeneration in chronic wound. To achieve rapid and effective wound healing, different types of wound dressings based on hydrocolloids, hydrogels, foams, and films 
have been developed, while incorporating bioactive growth factors, drugs, and cells.

In the current literature, Paula T. Hammond and colleagues coated woven nylon contact layer dressings with $V E G F$ - and $P D G F-B B$-loaded multilayer films by using a layer-by-layer (LbL) process (Fig. 4, top panel). The multilayer films were composed of repeating layer architectures consisting of poly( $\beta$-amino esters) (poly 1 and poly 2), poly(acrylic acid) (PAA), heparin sulfate (HS), VEGF, and/or PDGF-BB. The degradation rate of the overall film was controlled by poly( $\beta$-amino esters) and active growth factors were incorporated into PAA and HS. To prevent interlayer blending while releasing PDGF-BB, highly crosslinked barrier layers were added through chemical conjugation of cysteine to PAA. From the in vitro cellular assays measuring PDGF receptor $\beta$ phosphorylation level and migration level of primary human dermal fibroblasts (HDF), growth factors incorporated into multilayer films were verified to be active.
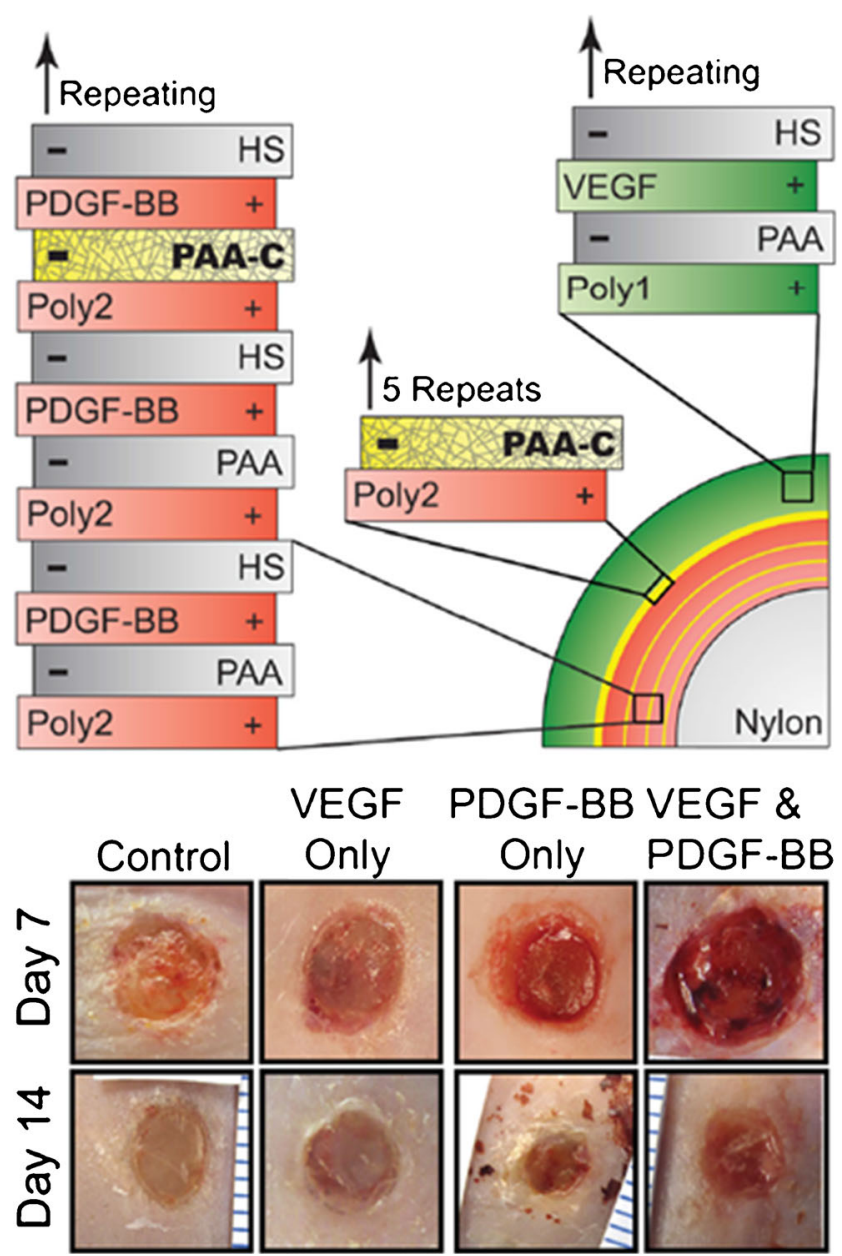

Fig. 4 Structure of wound dressing with $V E G F$ - and $P D G F-B B$-loaded multilayer films (top) and images of wounds after different growth factor treatments (bottom). Reproduced with permissions [28]. Copyright 2015, John Wiley \& Sons, Inc.
When VEGF/PDGF-BB combined dressing was covered on a chronic wound made on the back of $\mathrm{a} d \mathrm{db} / \mathrm{db}$ mouse, which is a genetic model of type II diabetes, it induced a higher density of blood vessel formation compared to VEGF-only-treated wounds and PDGF-BB-only-treated wounds (Fig. 4, bottom panel). Particularly strong redcolored granulation tissue was generated in VEGF/PDGFBB-treated wounds, indicating that healthy vascularized tissue was formed. From the histological analysis, only VEGF/PDGF-BB combined dressing demonstrated significantly increased amounts of newly formed granulation tissue within the wound sites among all VEGF-only-treated, PDGFBB-only-treated, and untreated groups, at 1 week after treatment. Cellular proliferation levels around the wound site were highest in VEGF/PDGF-BB-treated wound sites as compared to the other treated groups. After another week, this granulation tissue completely filled the wound site, whereas wound treated with PDGF-BB or VEGF resulted in partially generated granulation tissue.

Hammond and colleagues established a new therapeutic strategy of delivering multigrowth factors for chronic ulcerations, which can improve translational effectiveness while reducing dosage levels. A growth-factor-releasing multilayered platform can be easily incorporated into commercially available wound dressings for a reasonable cost. Also, this flexible multilayer growth factor delivery platform can be extended to treat other tissues [31]. Taken together, future development of the combinatory growth factor release platform will lead to a higher level of understanding and control in regard to the complex secretion mechanism of growth factors for tissue regeneration.

\section{Limb Regeneration by Decellularized Multitissue Scaffold}

\section{A limb graft made from decellularization and recellularization of the musculoskeletal system can maintain its intrinsic architecture and biomechanics, facilitating muscle-like function, blood perfusion, and in vivo transplantation}

Regenerative engineering aims to repair highly orchestrated multitissues, through synergetic integration of tissue engineering with advanced material science, stem cell science, and developmental biology [1]. Like the limb generation of a salamander, regenerative engineering pursues complete restoration of a lost body part, which involves a complex association of different tissues. Here, we introduce an innovative engineering approach to create a bioartificial limb graft, which is transplantable in vivo. 
In the USA, more than 1.5 million people are living with limb loss which requires prosthetic devices or reconstructive surgeries. However, while prosthetic devices are still limited with their functions, patients treated by reconstructive surgeries suffer from side effects and long-term immunosuppression. To overcome previous limitations in reconstructive therapies, writing in Biomaterials, Harald C. Ott and his colleagues from Harvard Medical School and Massachusetts General Hospital reported regeneration of a functional musculoskeletal system by utilizing decellularized native extracellular matrix (ECM) scaffolds and repopulating multiple types of cells, composed of myogenic, vascular, and mesenchymal cells [32]. This bioartificial composite tissue graft contained all bone, muscle, tendon, and nerve tissues, while maintaining its intrinsic architecture and mechanical strength. In addition, when this bioartificial composite tissue graft was transplanted orthotopically in a rat model, blood perfusion was observed through a vascular tree (Fig. 5).

To heal severely damaged organs, the treatment strategy has been extended from the repair of individual tissues to the regeneration of a multitissue system [33, 34]. At the interface of different tissues, spatial regulation of stem cell differentiation and selective positioning of tissue-specific cells are the major factors to successfully generate complex organ functions. In this respect, the design of a bioartificial organ construct is important to organize different types of cells into proper positions. To build sophisticated architectures of bioartificial organs, various approaches have been developed, including 3D bioprinting, organoid engineering, and whole-organ decellularization and recellularization [34]. 3D bioprinting can generate patterned scaffolds through layer-by-layer additive fabrication, while aiming to print more complex and large tissue architectures with high resolution. In addition, organoid engineering can create mini-organs from the differentiation of stem cells in 3D condition, while challenging to achieve clinically relevant scales. Finally, whole-organ decellularization and recellularization can recapitulate $3 \mathrm{D}$ organ architectures
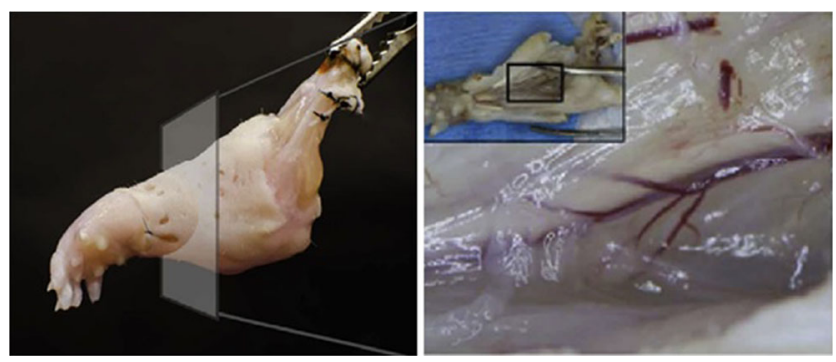

Fig. 5 Photo image of a regenerated limb graft (left) and re-perfused vascular tree (right) (reprinted from Figs. 3 and 4 in Jank et al. [32] with permission from Elsevier; copyright 2015, Elsevier Ltd) and thus can lead different types of cells to be delivered into their physiological locations. As a result, acellular ECM scaffold platforms have been widely applied to regenerate heart, lung, kidney, and limb [32, 35-37]. Currently, this strategy focuses on the enhancement of target-specific cellular delivery and engraftment with surrounding body systems.

Harald C. Ott and his colleagues are one of the leading research groups that are developing decellularized organ scaffolds for regenerative engineering [38]. To obtain a decellularized limb graft, rats were euthanized, heparinized, and dissected to isolate the forearm. After sufficient flushing, the forearm was mounted on the organ chamber to apply perfusion and to wash out residual debris. For the recellularization, decellularized rat forearms were placed in a bioreactor, which can perfuse a medium through a closedcircuit system, and muscle tissue, mouse skeletal myoblasts (C2C12), mouse embryonic fibroblasts, and primary human umbilical vein endothelial cells (HUVECs) were seeded by injection. Also, electrical stimulation was applied to the engineered graft to enhance alignment of cells and thus to form a functional muscle tissue. Finally, skin grafts harvested from donor rats were transplanted onto the engineered forearm graft using sutures and fibrin glue.

The decellularized musculoskeletal scaffolds were able to preserve tissue architectures, mineral densities, and mechanical properties, in a similar level to native tissue. In addition, vascular channels were highly connective throughout the entire forearm grafts. After transplantation of limb grafts into rats, anastomosis between brachial vessels of grafts and axillary vessels of rats facilitated re-perfusion of blood through connected vasculature. In addition, when electrical stimulation was applied, wrist and metacarpophalangeal joints of the limb graft performed flexible movements similar to those of native tissues, demonstrating their feasibilities in conducting muscle-like functions.

Through the innovative decellularization and recellularization strategy, Harald C. Ott and his colleagues demonstrated that the regenerated limb graft was able to maintain its biological functions and transplantable in vivo. This approach extended the previous single-tissue level of decellularized scaffolds into complex acellular scaffolds, containing multitissues in their physiological context. Another remarkable feature of the decellularized scaffold platform is that it can be extensively applied for the regeneration of other complex tissues as well, by positioning proper organ-specific cells. Currently, long-term stability, easiness of supply, and improvement in bioperformance are the remaining issues for the enhancement of acellular scaffolds. Once these limitations are overcome, the decellularized scaffold platform will be one of the most powerful tactics for regenerative engineering, broadening the concept of regeneration engineering from healing of multitissues to creation of multitissues. 
Acknowledgments For this News and Views edition, we acknowledge the helpful contributions of Hae Lin Jang and Katelyn Brinegar, Harvard Medical School.

\section{References}

1. Laurencin C, Khan Y. Regenerative engineering. Sci Transl Med. 2012;4, 160ed169.

2. Yan $\mathrm{R}$ et al. Nanowire-based single-cell endoscopy. Nat Nanotechnol. 2012;7:191-6.

3. Shalek AK et al. Vertical silicon nanowires as a universal platform for delivering biomolecules into living cells. Proc Natl Acad Sci. 2010;107:1870-5.

4. Robinson JT et al. Vertical nanowire electrode arrays as a scalable platform for intracellular interfacing to neuronal circuits. Nat Nanotechnol. 2012;7:180-4.

5. Chiappini $\mathrm{C}$, et al. Biodegradable silicon nanoneedles delivering nucleic acids intracellularly induce localized in vivo neovascularization. Nat Mater. 2015.

6. Chiappini $\mathrm{C}$ et al. Biodegradable nanoneedles for localized delivery of nanoparticles in vivo: exploring the biointerface. ACS Nano. 2015;9:5500-9.

7. Prausnitz MR, Langer R. Transdermal drug delivery. Nat Biotechnol. 2008;26:1261-8.

8. Prausnitz MR, Mitragotri S, Langer R. Current status and future potential of transdermal drug delivery. Nat Rev Drug Discov. 2004;3:115-24.

9. Prausnitz MR. Drug delivery: puncturing cells en masse. Nat Mater. 2015;14:470-1.

10. Undevia SD, Gomez-Abuin G, Ratain MJ. Pharmacokinetic variability of anticancer agents. Nat Rev Cancer. 2005;5:447-58.

11. Olive $\mathrm{KP}$ et al. Inhibition of Hedgehog signaling enhances delivery of chemotherapy in a mouse model of pancreatic cancer. Science. 2009;324:1457-61.

12. Huang B et al. Active targeting of chemotherapy to disseminated tumors using nanoparticle-carrying T cells. Sci Transl Med. 2015;7: 291ra294-4.

13. Peer $\mathrm{D}$ et al. Nanocarriers as an emerging platform for cancer therapy. Nat Nanotechnol. 2007;2:751-60.

14. Farokhzad OC, Langer R. Impact of nanotechnology on drug delivery. ACS Nano. 2009;3:16-20.

15. Su Y, Xie Z, Kim GB, Dong C, Yang J. Design strategies and applications of circulating cell-mediated drug delivery systems. ACS Biomater Sci Eng. 2015;1:201-17.

16. Kaiser A et al. Towards a commercial process for the manufacture of genetically modified $\mathrm{T}$ cells for therapy. Cancer Gene Ther. 2015;22:72-8.

17. Stephan MT, Moon JJ, Um SH, Bershteyn A, Irvine DJ. Therapeutic cell engineering with surface-conjugated synthetic nanoparticles. Nat Med. 2010;16:1035-41.

18. Driessens FC, Verbeeck R. Biominerals. CRC Press; 1990.
19. Jang HL et al. Revisiting whitlockite, the second most abundant biomineral in bone: nanocrystal synthesis in physiologically relevant conditions and biocompatibility evaluation. ACS Nano. 2013;8:634-41.

20. Jang HL, et al. In vitro and in vivo evaluation of whitlockite biocompatibility: comparative study with hydroxyapatite and $\beta$ tricalcium phosphate. Adv Healthcare Mater. 2015;n/a-n/a. doi: 10.1002/adhm.201400824.

21. Jang HL et al. Phase transformation from hydroxyapatite to the secondary bone mineral, whitlockite. J Mater Chem B. 2015;3: 1342-9.

22. Meinke D, Skinner H, Thomson K. X-ray diffraction of the calcified tissues in Polypterus. Calcif Tissue Int. 1979;28:37-42.

23. Quint P, Althoff J, Höhling H, Boyde A, Laabs W. Characteristic molar ratios of magnesium, carbon dioxide, calcium and phosphorus in the mineralizing fracture callus and predentine. Calcif Tissue Int. 1980;32:257-61.

24. Fonder MA et al. Treating the chronic wound: a practical approach to the care of nonhealing wounds and wound care dressings. J Am Acad Dermatol. 2008;58:185-206.

25. Falanga V. Wound healing and its impairment in the diabetic foot. Lancet. 2005;366:1736-43.

26. Medina A, Scott PG, Ghahary A, Tredget EE. Pathophysiology of chronic nonhealing wounds. J Burn Care Res. 2005;26:306-19.

27. Blakytny R, Jude E. The molecular biology of chronic wounds and delayed healing in diabetes. Diabet Med. 2006;23:594-608.

28. Almquist BD, Castleberry SA, Sun JB, Lu AY, Hammond PT. Combination growth factor therapy via electrostatically assembled wound dressings improves diabetic ulcer healing in vivo. Adv Healthcare Mater. 2015.

29. Moura LI, Dias AM, Carvalho E, de Sousa HC. Recent advances on the development of wound dressings for diabetic foot ulcer treatment - a review. Acta Biomater. 2013;9:7093-114.

30. Boateng JS, Matthews KH, Stevens HN, Eccleston GM. Wound healing dressings and drug delivery systems: a review. J Pharm Sci. 2008;97:2892-923

31. Macdonald ML, Rodriguez NM, Shah NJ, Hammond PT. Characterization of tunable FGF-2 releasing polyelectrolyte multilayers. Biomacromolecules. 2010;11:2053-9.

32. Jank BJ et al. Engineered composite tissue as a bioartificial limb graft. Biomaterials. 2015;61:246-56.

33. Hutmacher DW. A road map for a tissue engineering concept for restoring structure and function after limb loss. J Mater Sci Mater Med. 2013;24:2659-63.

34. Ren X, Ott H. On the road to bioartificial organs. Pflugers Arch Eur J Physiol. 2014;466:1847-57.

35. Ott HC et al. Perfusion-decellularized matrix: using nature's platform to engineer a bioartificial heart. Nat Med. 2008;14:213-21.

36. Song JJ et al. Regeneration and experimental orthotopic transplantation of a bioengineered kidney. Nat Med. 2013;19:646-51.

37. Ren $X$, et al. Engineering pulmonary vasculature in decellularized rat and human lungs. Nat Biotechnol. 2015.

38. Guyette JP et al. Perfusion decellularization of whole organs. Nat Protoc. 2014;9:1451-68. 